the operative interference with the metacarpal joints must not be neglected. The due mobility of these transverse arches is also of importance in picling up objects, as without it, although opposition of the thumb may be free and un. impaired, the thumb cannot reach farther than the middle finger, and the fourth and fifth fingers cannot be used in conjunction with it. Abduction and opposition of the thumb are easily lost after septic wounds of the palm. If opposition is defective a band of adhesive strapping round the hand, keeping the thumb in opposition and preserving the palmar arches, reapplied as progress is made and the band loosens, is quite effective. For deficiency in abduction a series of small plaster splints moulded to the first interdigital space will gradually restore function.

Fingers may become stiff from a variety of causes, but assuming them to be potentially mobile and useful-that is to say, that all the structures essential to their mobility are present-their restoration to function is a purely mechanical problem. In dealing with the problem elsewhere $\mathrm{I}$ described certa:n methods of treatment by metal splints and plaster that I had devised. I still use these methods and find them effective, but others have published other devices equally good, and the principles involved are in the main identical. If a masseuse move a stiff finger to and fro repeatedly, resistance is induced, often unnecessary pain, and a certain amount of reactionary inflammation. Should massage alone without mechanical aid be employed, the hand should have a preliminary bath treatment, preferably paraffin, and the massage immediately after should be confined to a single movement of the affected joint in each direction to the full extent possible without pain; the patient should then be encouraged and re-educated in active movements. I strongly disapprove of forcible mobilization of stiff fingers under an anaesthetic; though applicable to other joints, this method is not satisfactory for the fingers, and more gradual methods are indicated. The classification and the principles of treatment I adrocate are as follows:

A. Fingers flexed when wrist is dorsiflexed, extension possible with wrist flexed. This, the type of ischaem:c contraction due to shortening of flexor tendons, is best treated by Sir Robert Jones's method, the joints being straightened seriatim, beginning distally, leaving each joint in the deformed position until all the joints distal to it have been corrected, and retaining that correction by continued splinting, ending up with dorsiflexion of the wrist.

B. Wrist movement free, ove or more fingers flexed at all three joints, contraction unaffected by position of wrist. One must aim at obtaiuing extension without losing flexion. I employ a metal splint extending beyond the fingers and terminating in a bar, fixed to the hand by plaster. 'To the affected fingers loops of adhesive plaster are fixed and tapes are tied between these and the bar. By gradual tightening of these tapes extension is obtained, but every day the tapes are released and the finger is brought back to its original flexed position and the tape then retied. There is then no loss of flexion; should this be noticed, extension is being carried out too rapidly and one must call a halt.

C. Free movement at the metacarpo-phalangeal joint, distal joints flexed. Gradual straighteuing on metal palmar gutter splints will suffice, flexion being preserved by daily movements, as before.

D. Metacarpo-phalangeal joints stiff in hyperextension, distal joints straight or slightly tlexed. The important principle in treatment consists in getting the metacarpo-phalangeal joints to pass the dead point or straight line position. For this condition I use an ordinary short cocli-up splint (which keeps the wrist dorsiflexed) with attached to it a quadrilateral wire loop springing from its palmar surface at the wrist and extending to the level of the metacarpal extremities. To this the fingers are flexed by tapes; daily movement as before.

These methods exert very great force, but can be graduated to a nicety; correction is gradual and gives no pain, and the range of movement is never lost. Tape traction is better than elastic, as there is no tendency on the part of the patient to resist it. Finally, employment of the hand, at first in gymnastic exercises and later in actual work, will put the tinishing touches on the treatment.

\section{RE FERENCES.}

1 Sir Robert Jones: Injuries to Joints. Oxford War Primers. 2 Robert 13. Osgood: Radio.humeral Bursitis, Epicondylitis, Epicondylalgia (Tennis Elbow). ${ }^{3}$ An Abnormality of the Tendon Grooves of the Radius British Medicat Jorrial, May 10th, 1919. I Stiff Fingers, with Special Reference to Methods of Treatment by Metal and Plaster Splints, Journ. of Orthop. Surgery, June, 1919.

\section{THE DOSAGE OF RADIUM.}

$$
\text { BY }
$$

DAWSON TURNER, M.D.EDIN.,

IN CHARGE OF THE RADIOM TREATMENT AT EDINBURGH ROYAL

$$
\text { INFIRMARY. }
$$

ThE duration of the application is a most important element in radium treatment, and it is one which there may be a tendency to overlook in institutions where large quantities of radium are available. Consider, for an example, a dose of $500 \mathrm{mg}$. hours, which would be a fair dose to administer to a small rodent ulcer. This dose might be given by $10 \mathrm{mg}$. of radium for fifty hours, or by $50 \mathrm{mg}$. for ten liours, or by $500 \mathrm{mg}$. for one hour. Does anyone contend that similar effects would be produced in each case? I have no hesitation in making the affirmation, based upon an experience of twenty years, that for cach case there is a certain optimum combination of milligrams and of hours, and that this question is or:e for the radium expert to decide. Levin and Joseph, ${ }^{1}$ from an experimental study of the influence of radiations on the development and growth of the crown gall on plants, have shown. that the primary effect of radium or $x$-ray radiations consists in the inlibition of the proliferating power of the cancer. cells and not in their direct destruction. The crown gall, tumour-like formation on plants is so called because the growth frequently appears where stem and root join (called the crown). It is due to a parasite and can be induced artificially by inoculating a plant with an agar culture of the micro-organism. Plants were inoculated by these observers and some were submitted to radiation and some kept as controls; the control plants developed large crown galls, the others none or stunted growths containing true tumour cells. Cancer cells go through a cycle of (1) youth, (2) maturity, (3) old age. At one time they are actively dividing and at another they are not, and it has been shown that they are more susceptible to radiations when their nuclei are dividing than when they are in any other stage. In certain stages they may be as immune as normal tissues.

The lesson to be learned from a consideration of these facts is that in order that the dose of radium may be effectual it must be applied for a certain time, for it is only by a pro. longed application that we can make fairly sure of catching all the cancer cells in their susceptible stage. We may therefore conclude that small amounts of radium for prolonged periods are more likely to prove beneficial than large amounts for'short periods. 'The oven may be too hot and tho - bread will be burned on the outside instead of being baked throughout.

To take another point: suppose one possesses $100 \mathrm{mg}$. of radium, in what quantities is it best to put it up into tubes? The answer depeuds mainly upon the nature of the diseased condition to be attacked, upon its situation, its area, its volume, and upon whether the radium is to be applied externaliy or buried in the growth. Take the case of a lymphosarcoma -perhaps the most susceptible of all growths to radium-of the size of a large orange; in this case it would be best to have the radium put up in 5 or $10 \mathrm{mg}$. portions in separate tubes, so that they could be distributed throughout the mass, and particularly arranged like sentinels around the borders of the growth, for in this way the central parts will be receiving cross-fire radiation from all the tubes, but the peripheral parts will only get efficient radiation from the peripherally placed tube which happens to be nearest. In order that the whole of a growth may be efficiently and uniformly radiated the radium should theoretically be uniformly distributed throughout it-in fact, tho desideratum would be to scatter radium like pepper from a pepper pot throughout the mass. Though this is for several reasons impracticable yet the more nearly we can approach to it the better. Dr. Stevenson's needles are a means to this end. Small tubes are filled with the radium emanation and are enclosed in hypodermic needles for embedding in the neoplasm. There is a disadvantage which applies to all cases in which the emanation is used, owing to its steady loss of strength-namely, that the dosage is not known unless the tubes have been tested for their emanation strength before being employed and unless a table of their rate of decay be consulted. As the time element in radium treat: ment, as I have endeavoured to show, is important, emanation tubes have the disadrantage that they would have to be replaced from time to time by freshly cliarged ones. Where the application is external this would be easily done, but in 
the case of a tumour, in which the radium is embedded, it would mean a minor surgical operation each time the emanation tubes were changed.

Another point of importance is to give the whole maximum dose at first, and not to break it up into smaller ones which are repeated. The latter course may in certain cases turn out a fatal error, and this chiefly because the malignant cells, which were stunned but not killed by the first application, will, when they recover, tend to breed cells which are more immune to radiation, until, the same process continuing after several exposures, only those cells will survive which are refractory to the rays. There is a tendency by natural selection to breed cells which are immune, and further exposures will now only do harm. The knock-out blow should be given by a sufficiently long exposure to a sufficient amount of radium while the cancer cells are not suspecting an attack; they must be taken by surprise, so that they will be unable to organize a resistance and be incapable of putting up a serious defence.

Were more attention paid to these points there would, I venture to think, be fewer failures following radium treatment.

Rfference

1 Annals of Surgery, vol. 67, p. 442.

\section{THE FUNCTION OF THE ADRENAL GLANDS AND ITS RELATION TO CONCEN'TRA- TION OF HYDROGEN IONS.:}

BY

ROBERT McCARRISON, M.D., F.R.C.P.,

LIEUTENANT-COLONEL, INDIAN A EDICAL SERVICE; IN CHARGE OF THE DEFICIENCY DISEASES INQUIRY, INDIAN RESEARCH FUND ASSOCIATION.

(From the Pasteur Institute of Scuthern India, Coonoor.)

THE object of this research was to inquire further into the causation of the adrenal hypertrophy occurring in inanition and in avitaminosis. ${ }^{1}$ This hypertroplyy is accompanied by an increased epineplirin content of the glands, ${ }^{1}$ and is dug, in the avitaminosis of birds, to the absence of vitamins $A$ and $B$ from the food. It is associated also, as shown by Cramer, ${ }^{2}$ with an almost complete dis appearance of lipoids from the adrenal cortex. The enlargement rapidly reverts to a more nornal state when the missing vitamins are supplied. The question as to why hypertrophy of the adrenal glands should occur under conditions tlat give rise to greater or lesser degrees of atrophy of all other organs (the pituitary body in males excepted) ${ }^{1}$ is of fuudamental importance both in relation to the dis. orders of nutrition in which it is found and to the function of the adrenal b o dies themselves. The facts suggest some purpose underlying their enlargement; some purpose, also, in their increased content of epineplurin. It was thought that it might be related to the increased con centration of hydrogen ions that occurs in avitaminosis. Recently Kato, 'Takalira, and others have drawn attention to the increased bydrogen ion concentration of nerves, spinal cord, heart, and blood ressels in poly. neuritis avium. I have con. firmed this observation in so far as it relates to the heart, and found also an increase of $\mathrm{P}_{\mathbf{H}}$ concentra. tion in pectoral muscle. In a few cases of poly. neuritis columbarum accom panied with effusion into the pericardial sac or with oedema of tlie tissues, the dropsical fluid has been well on the acid side of neutrality-pericardial effusion, for example, having a $\mathrm{P}_{\mathrm{H}}$ of approximately 6.5 . * Abstract of Twelf th Report to the Indian Research Fund Association
on the Pathogenesis of Deficiency Disease. The full puper will appear in an early number of the Indian Journal of Medical Research.
Experiments were accordingly undertaken to ascertain the effect of changes in concentration of hydrogen ions on the action of commercial adrenaline hydrochloride and of the active principle of the adrenal medulla-epinephrin-in various media.

Enucleated eyes of the toad (Bufo melanostictus) were employed for the purpose; in all 1,300 were uscd. Each test on one eye was controlled by the other eye of the same animal. It has been stated that while adrenaline causes mydriasis of the enucleated frog's eye, it does not do so in the enucleated toad's eye. So far as Bufo melanostictus is concerned this is not the case. It has long been known that, by its action in stimulating the dilator mechanism of the iris, adrenaline causes mydriasis; but, so far as I can find, no

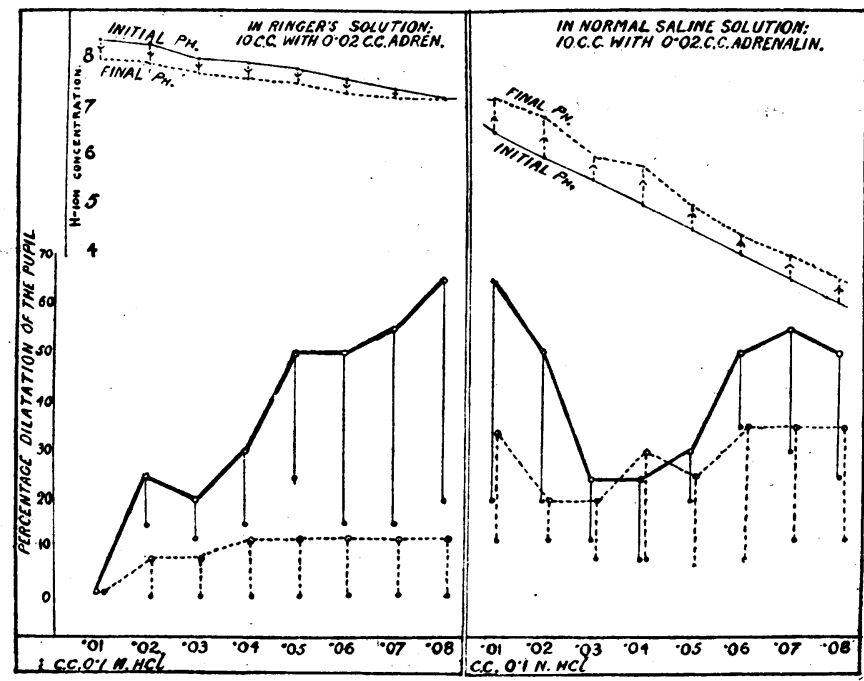

Fig. 1.-Showing the $f$ ffect on the action of adrenaline bydrochlorid adding increasing enucleated eye HCl to Ringer's solution 002 c.cm. of the commer containin of adrenaline ( 1 in 1, COJ). As the PH of the medium falls from alkalin:ty tonards neutrality th curre of maximum dilatation of the pupils (shown as a thick black line) gradually heightens, and the rang shown as vertical continued line falling from the points of maximum di'atation to points of maximum contraction). The curve of maximum dilatation and the ranges of action of the irides of control eyes subjected to the influence of the same dose of adrenaline, but without the addition are shown in amounts of $\mathrm{N} / 10 \mathrm{HCl}$ approximate inilial and final $P H$ in t'e tubes to which HCl was auded are shown at the top of the figure. ipproxim of control tubes was that under the influence of the metabolic activities of the eye the $\mathbf{H}$ falls towards neutrality durin the course of the experiment (thirty the course of the experiment (thirty

FIG. 2.- Showing the effect on the ction of adrenaline hydrochloride of adding increasing quantities of $\mathrm{N} / 10 \mathrm{HCl}$ to normal saline solution containing $0.02 \mathrm{ccm}$. of the com(1 in 1,000). As the acidity increases the curve of maximnm diletation an 1 the rance of action of the irides point of acidity is reached at $0.03 \mathrm{c.cm}$. N/10 HCl. The lesser degrees of acidity $(0.01$ and 0.02 $\mathrm{N} / 10 \mathrm{HCi}$ enhivce the action of adrenaline on the papil of the enuclester ere: greater degree $(0.03$ to $0.5 \mathrm{~N} / 10 \mathrm{BCl}$ ) inhibit its action; greater degrees still enhance a gain, while the irides tend to he fixed in the position of mydriasis. The gulf of inhibition is wider in normal saline than in serum (compare Fig. 3). A pproximate initia and tinal $\mathrm{PH}_{\mathrm{H}}$ in acid tubes are shown at the top of the flgure; initial $\mathbf{P H}_{\mathbf{H}}$ of control tubes was approximalely 7 . It will be noted that under the influence of the metabolic activity of the eyes the $\mathbf{P H}$ in the acid tubes rises towards neutrality. Experimental eyes, uninterrupted lines: controls, without acid, dotted lives. Duration of observation, thirty minutes. approximately 8 . It will be noted drop precipitately until the critical reference is macle in the literature to its further and, as I belicve, more imporlaut action of sensitizing the enucleated eye, in such a way that tho iris respond more readily to the norua stimuius of light through its maximum rauge of action. 'This sensitivity of the toad's iris to the stimulus of light. when under the influence of adrenalino hydrochloride or of epinepluriu, was utilized as a means of determining the effect upon the action of these substances of chances in concentration of liydrog( 1 jons in various media. $\mathrm{PH}_{\mathrm{H}}$ indicator lablets ("A. and $H: ")$ were used through. out; the PH values are therefore approximate.

The observations made in the course of the inquiry are enumerated below; thic reader is referred to the full paper for details regard. ing them.

1. There is in the eye of Bufo melanostictus a peri. pheral mechanism which can act independentiy of tho nerve centres. It is respon. sive to the stimulus of light within a limited range-25 per cent. dilatation in the dark to slit-lilie contraction on exposure to light-pro. vided the eye be preserved in media of suitable composition and suitable $\mathbf{P H}$ concentration. In such it retains a gradually waning sensitivity for a compara. tively short period-approximately two liours.

2. 'This mechanism can be so sensitized by ad. renaline hydrochloride or by eninephrin that, under appropriate conditions, the iris will respond with enhanced sensitiveness to the stimulus of light through its maximum range of action. It retains its enhanced sensitivity for prolonged periods-three to eight hours-and in exceptional cases for even Jongev periods. This effect of adrenaline and of epinephrin is spoken of as "the adrenaline-iris-light-sensitivity reaction." 